\title{
OTIMIZAÇÃO NA UTILIZAÇÃO DE ÁREAS INTERNAS EM UMA INDÚSTRIA AUTOMOTIVA ATRAVÉS DA UTILIZAÇÃO DO ALGORITMO FIRST-FIT DECREASING
}

\author{
Alana Paulatti Peri ${ }^{1}$, Maria Teresinha Arns Steiner ${ }^{1}$ e Ricardo de Almeida ${ }^{1}$ \\ ${ }^{1}$ Pontifícia Universidade Católica do Paraná - PUCPR \\ E-mails: alana.paulatti@gmail.com, maria.steiner@pucpr.br e \\ ralmeida80@hotmail.com
}

\section{RESUMO}

A crise econômica brasileira que atinge a indústria automotiva já reduziu, de janeiro a abril de 2016, 25,41\% da produção de veículos, se comparado ao mesmo período do ano anterior. Já as vendas de automóveis foram impactadas em $27,63 \%$, no mesmo período analisado. Diante disso, inúmeras indústrias, com o intuito de aumentar a produtividade e reduzir o número de atividades que não geram valor agregado ao produto final, têm buscado otimizar seus processos e meios de produção. No caso da empresa analisada, objetivou-se otimizar as áreas internas, conhecidas como áreas de kitting, através de técnicas de otimização aplicadas aos problemas do tipo Bin Packing Problem (BPP) unidimensional. Aplicou-se o algoritmo de aproximação First-Fit Decreasing (FFD) em 20 áreas de kitting, com o intuito de criar uma ferramenta que permita a definição da quantidade ótima flowracks, meio logístico tubular que apresenta três prateleiras, necessária para a alocação das embalagens das peças. Assim, foi possível obter uma redução total de 23 flowracks, representando uma diminuição de 17,16\% nas quantidades necessárias, e ganho de $89,36 \mathrm{~m}^{2}$ de área livre total nas áreas de kitting.

\section{INTRODUÇÃO}

A crise econômica brasileira afetou, de janeiro a abril de 2016, 25,41\% da produção de veículos, se comparado ao mesmo período do ano anterior, de acordo com o relatório anual da Associação Nacional dos Fabricantes de Veículos Automotores (ANFAVEA) [1]. Dentro deste universo, segundo a Federação Nacional da Distribuição de Veículos Automotores (FENABRAVE) [2], as montadoras automotivas sofreram um impacto de $27,63 \%$ de redução nas vendas de automóveis, na comparação entre os dois períodos.

Diante disso, inúmeras indústrias têm buscado otimizar seus processos e meios de produção, com o intuito de aumentar a produtividade e reduzir o número de atividades que não geram valor agregado ao produto final. Um exemplo disso no caso específico da Empresa $\mathrm{ABC}$, indústria abordada neste estudo, é a existência de áreas intermediárias, 
denominadas de áreas de kitting (AK), dedicadas ao armazenamento de peças, além da formação de conjuntos de peças e do envio destes à linha de montagem principal.

Nestas áreas, as embalagens que apresentam dimensões superiores a $1.000 \mathrm{~mm}$, são armazenadas no solo, e as inferiores a $1.000 \mathrm{~mm}$, devem ser armazenadas em móveis gravitacionais tubulares com três prateleiras (flowracks). Dessa forma, a concepção destas áreas é diretamente proporcional ao número de referências distintas de peças existentes, e a otimização se torna uma alternativa viável na consideração de ocupação de superfícies produtivas.

Sempre que é lançado um novo projeto automotivo na empresa estudada, ocorrem duas situações problemáticas: a falta de ferramentas padronizadas para a determinação da real necessidade de aquisição de novos flowracks; e, o impacto da não utilização de ferramentas para otimizar o espaço consumido por AK.

Todas as atividades relacionadas a esses dois fatores são realizadas de acordo com o conhecimento prévio dos analistas. A título de exemplo, pode-se citar o seguinte: para cada inserção de embalagem de peça em um flowrack, é necessário o encaixe a "olho nu". Assim, cada modificação implica em um retrabalho grandioso, já que é preciso realocar todas as demais referências de peças já existentes. Atualmente, 1.357 referências distintas de peças já estão alocadas em 136 flowracks. Com a entrada dos novos veículos, por exemplo, essa demanda subiu para 2.099 referências.

De posse dessas informações, neste trabalho objetiva-se determinar a quantidade ótima de flowracks, através de um algoritmo de otimização conhecido como First-Fit Decreasing (FFD) aplicado aos problemas do tipo Bin Packing Problem (BPP) unidimensional, a fim de seja possível otimizar as AKs.

O presente artigo está organizado da seguinte forma: na seção 1 é descrita resumidamente a revisão da literatura, ou seja, o problema do Bin Packing; a formulação matemática para este tipo de problema; a descrição do algoritmo FFD; além de alguns trabalhos correlatos; na seção 2, tem-se a descrição da empresa bem como 0 detalhamento do problema abordado; já na seção 3, detalha-se a metodologia aplicada; na seção 4, os resultados são apresentados; por fim, na seção 5, são apresentadas as conclusões.

\section{BIN PACKING PROBLEM (BPP)}

O termo bin packing foi introduzido por Johnson, em 1973, em sua tese de doutorado intitulada Near optimal bin packing algorithm, a fim de substituir o termo loading quando se aborda problemas de designação de arquivos de dados de determinados comprimentos às trilhas de um disco rígido [3]. Assim, iniciou-se a denominação de bin packing, variantes ou generalizações, a assuntos relacionados a área de scheduling, assembly line balancing, alocação de memória e Cutting Stock Problem (CSP). 
O BPP é definido por [4] como a necessidade de se alocar um conjunto de itens em um número mínimo de grandes objetos (bins), de capacidades idênticas. Assim, é dado um número inteiro positivo $C$ e um conjunto $N=\{1, \ldots, n\}$ de itens, cada qual com dimensão inteira e positiva $w_{i}(i=1, \ldots, n)$ que satisfaça $w_{i} \leq C$. O BPP consiste em obter o menor $m$ inteiro tal que exista uma partição $B_{1}, B_{2}, \ldots, B_{m}$ do conjunto $N$ em que o somatório $w\left(B_{j}\right)=\sum_{w_{i} \in B_{j}}^{j=1, \ldots, m}$ dos pesos dos itens em cada subconjunto $B_{j}(j=1, \ldots, m)$ não exceda C.

De acordo com a tipologia de Dyckhoff [5], o BPP é um problema do tipo 1/V///M, conhecido por se tratar de um problema NP-hard. Foi argumentado por [3] e [7] que problemas desta classe são intratáveis, de forma que se torna improvável a existência de um algoritmo de otimização exata que seja capaz de abranger toda e qualquer instância em tempo polinomial ou pseudopolinomial, a menos que $P=N P$.

O BPP possui diversas aplicações práticas e cotidianas. Alocações de arquivos, ordenação de atividades independentes, carregamento de caminhões com restrições de peso, e inserção de anúncios em intervalos comerciais na programação televisiva são exemplos dessas aplicações [3] e [8].

No trabalho de [9] é possível encontrar, também, outros exemplos de aplicação relacionadas a organização do trabalho na indústria: organização da produção de tal forma a garantir a máxima realização do plano sob condições de um determinado mix de produtos; distribuição de ordens de produção a máquinas com índices de produtividade diferentes; maximização da utilização de maquinário; minimização de refugos produtivos oriundos de processos de corte; melhor utilização de combustíveis, dentre outros. [10] reitera que, deste trabalho de Kantorovich, surgiu um dos principais modelos matemáticos para os problemas de BPP e CSP.

\subsection{Formulação matemática}

É fornecido por [11] uma possível formulação matemática para o problema, apresentada de (1) a (7), a seguir, em que $w_{j}=$ peso do item $j$ e $c=$ capacidade cada bin, atribui-se cada item a um bin, tal que o peso total dos itens não exceda a capacidade $c$ e que a quantidade de bins seja mínima.

$$
\begin{aligned}
& \min z=\sum_{\substack{i=1 \\
n}}^{n} y_{i} \\
& \text { s. } a \\
& \sum_{j=1}^{n} w_{j} x_{i j} \leq c y_{i}, \quad i \in N=\{1, \ldots, n\}, \\
& \begin{array}{ll}
\sum_{i=1}^{n} x_{i j}=1, & j \in N, \\
y_{i}=0 \text { ou } 1, & i \in N,
\end{array}
\end{aligned}
$$




$$
\begin{array}{rlrl}
x_{i j} & =0 \text { ou } 1, & i \in N, j \in N, \\
c \in \mathbb{Z}^{+} & \\
w_{j} \leq c, & j \in N
\end{array}
$$

onde, a função objetivo (1) busca obter a quantidade mínima de bins utilizados. A restrição (2) assegura que a capacidade de cada bin seja respeitada. A restrição (3) garante que o item $j$ seja atribuído uma única vez ao bin. Em (4) e (5), as variáveis $y_{i}$ assumem o valor " 1 ", se o bin $i$ for usado, e "0" caso contrário; e para $x_{i j}$ se atribui o valor "1", se o item $j$ for designado ao bin $i$, e "0" caso contrário. Se a restrição (6) for violada, $c$ pode ser substituído por $\lfloor c\rfloor$. Se algum item violar a restrição (7), então a instância é trivialmente inviável. Em qualquer solução viável, por questão de simplicidade, assume-se que os bins de menor índice são utilizados, isto é, $y_{i} \geq y_{i+1}$ para $i=1, \ldots, n-1$.

\subsection{First-Fit Decreasing (FFD)}

O algoritmo FFD faz parte da classe dos algoritmos de aproximação para resolução de BPP tidos como off-line. Para tanto, funciona com uma regra de préprocessamento de reordenação decrescente dos itens. É colocado por [7] que após esse processo, o FFD funciona da seguinte forma:

1. $P$ um empacotamento de uma lista $L$;

2. $j=M I N\left\{j^{\prime} \mid B I N_{j^{\prime}}\right.$ está usado em $P$ e $\left.\alpha_{j^{\prime}}+w_{i} \leq 1\right\}$; e

3. $w_{i}$ o próximo item de $\mathrm{L}$ a ser atribuído.

considera-se $\alpha_{j^{\prime}}$ como a capacidade do bin já utilizada e $w_{i}$ como o peso do item a ser atribuído, neste caso a capacidade do bin é igual a "1". Junqueira (2007), então, define a regra de empacotamento do FF, da seguinte forma:

- Se $\exists j$, como mencionado em 2, então atribua o $w_{i}$ ao $B I N_{j}$;

- Senão, abra um novo bin e atribua $w_{i}$ a este bin.

\subsection{Trabalhos correlatos}

Diante das inúmeras possiblidades de aplicação do BPP a problemas reais e atuais, é possível encontrar na literatura autores tais como [12], [13], [11], [4], [14], [15] e [16].

Foi proposto por [12] um procedimento exato baseado no algoritmo de enumeração implícita de Balas [16]. Na formulação proposta, a atribuição de pesos em cada bin foi realizada de forma que quanto maior o índice do bin, maior o peso. Assim, eliminase a necessidade de fazer uso de outras variáveis para indicar o uso de um bin, já que se assegura a utilização do menor número de bins. 
Ainda sobre BPP, [13] desenvolveram um método de solução em que utilizam uma combinação de enumeração implícita, caracterização de matrizes de alocação e várias regras que buscam evitar redundâncias de atribuições. Utilizaram, também, a Relaxação Lagrangeana para melhorar a eficiência do algoritmo.

Entretanto, ambos os métodos desenvolvidos são capazes de resolver apenas problemas de pequenas instâncias. Dessa forma, [11] propuseram o algoritmo MTP, baseado na estratégia de ramificação (branching) do FFD. [17] aponta a importância do trabalho de Martello e Toth [11] já que, ainda hoje, é referência básica para a resolução deste problema de forma exata.

Foi sugerido por [4] um problema gerador para o BPP para ser utilizado em avaliações futuras de métodos de solução exatos e heurísticos. Realizaram, também, alguns experimentos com o método FFD e o MTP a fim de identificar problemas difíceis para o BPP. Os autores confirmam a opinião geral de que o MTP representa o estado-da-arte para métodos exatos de solução para o BPP, ainda que o tamanho dos problemas, cuja resolução demande tempo computacional razoável, seja insatisfatório.

Já em 1997, [14] geraram um procedimento híbrido, chamado de BISON, para a solução exata do BPP através da combinação de bounds inferiores, procedimentos de redução tradicionais e novos, diversas heurísticas e a apresentação de um novo esquema de branching em um procedimento de branch-and-bound.

Em 2014, [15] combinaram as características das melhores heurísticas BPP, Algoritmos Genéticos de Agrupamento (GGA - Grouping Genetic Algorithm) e capacidades da computação paralela, para obter soluções ótimas de uma forma viável sem aumentar a comunicação entre os GGA, ao passo que se alcança maiores benefícios. Os GGAs híbridos e paralelos apresentados foram aplicados de forma satisfatória a 1.318 problemas de benchmark de BPP unidimensionais. A taxa de sucesso obtida com esses algoritmos é superior a maioria das abordagens encontradas na literatura, sendo capaz de alcançar a solução ótima em $88,54 \%$ das instâncias em tempos computacionais práticos.

Por fim, em 2016, [16] apresentaram um método exato, baseado na formulação arcflow com restrições laterais, para resolver o BPP e o CSP. O método inclui um algoritmo de compressão gráfica, que geralmente reduz substancialmente o grafo de base sem enfraquecer o modelo. A formulação proposta é equivalente a fornecida por Gilmore e Gomory [18], que proporciona uma relaxação linear forte. Entretanto, em vez de utilizar a geração de colunas no processo iterativo, o método constrói um grafo, onde cada "caminho" da origem até o nó de destino representa um padrão de empacotamento viável. Os autores conseguiram resolver a maioria das instâncias de benchmark, com uma média de menos de um minuto por instância. A maior limitação encontrada para o método é a combinação entre grandes capacidades e padrões longos, que podem se tornar difíceis de serem representados de uma forma compacta. 


\section{DESCRIÇÃO DO PROBLEMA}

A empresa analisada, com nome fictício de Empresa $A B C$, está entre as cinco maiores montadoras automotivas, no âmbito mundial. O complexo se localiza em São José dos Pinhais/PR, e é composto por três fábricas, responsáveis pela produção de veículos de passeio, utilitários e motores.

A unidade fabril escolhida é responsável pela produção de veículos de passeio, e possui quatro áreas de processos principais em sua cadeia produtiva: estamparia, onde chapas de aço são conformadas mecanicamente e transformadas em peças para a carroceria do automóvel; em seguida, a carroceria, em que as peças oriundas do processo anterior são soldadas e dão forma ao carro e, assim, o produto parcial é encaminhado à pintura; nesta fase, é adicionada a cobertura de tinta que fornece durabilidade e embelezamento ao veículo; por fim, inicia-se a montagem, na qual adiciona-se à carroceria previamente pintada todos os acabamentos internos e externos, bancos, vidros, motor, luzes, etc. Assim, ao fim de todas essas etapas que incluem, também, verificações e testes de qualidade, o veículo é considerado concluído.

\section{1 Áreas de kitting (AK)}

As AKs são definidas, dentro da empresa, como áreas destinadas ao agrupamento de itens e peças de uma mesma região (tronçon) da linha principal de montagem. Cada tronçon é responsável por realizar processos específicos como, por exemplo: tronçon responsável pela montagem do conjunto mecânico; tronçon responsável pela montagem de portas; tronçon responsável pela montagem de bancos e parachoques, etc. Portanto, para cada tronçon, exceto o tronçon 9, há uma área específica, que podem ser subdivididas em 20 AKs. A Figura 1 retrata essa relação. 
Figura 1 - As AKs e seus respectivos tronçons.

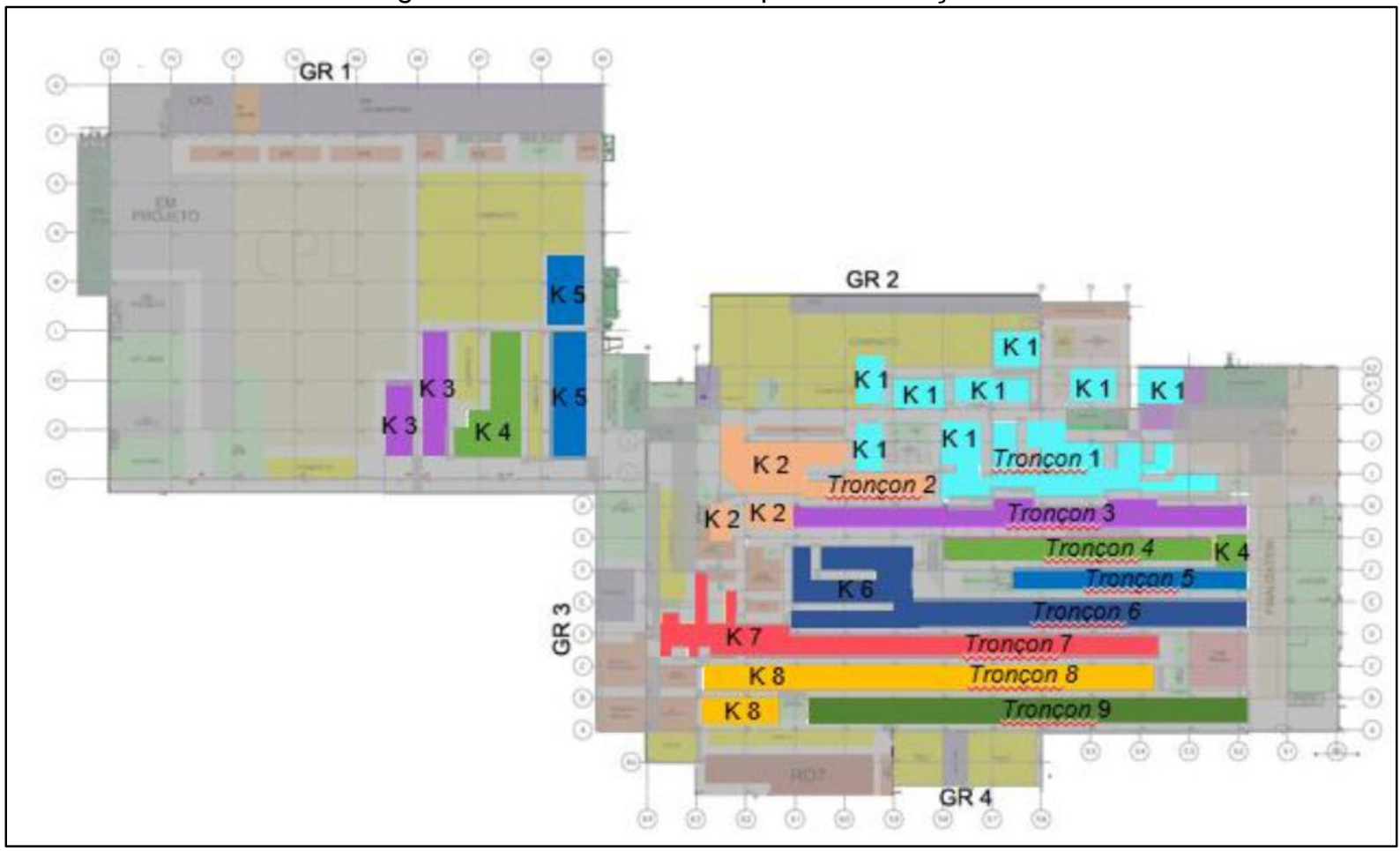

Fonte: Empresa ABC (2016).

De acordo com a "metodologia Kaizen" aplicada pela organização, a aplicação de AK objetiva atender a premissa de que os operadores que geram valor agregado ao produto, ou seja, realizam o processo real de montagem do automóvel, concentremse apenas nestas atividades, tanto no quesito movimentação física quanto no quesito cognitivo. Dessa forma, toda e qualquer atividade de valor não agregado nestes postos de trabalho, tais como caminhar para buscar peças, abaixar para alcançar itens em níveis inferiores, ou até mesmo o ato de pensar para escolher o que pegar, devem ser eliminadas ou, ao menos, minimizadas.

Assim, as AK envolvem a disposição de todas as referências de itens e peças de forma que os kitteurs - operadores responsáveis pela montagem dos "carrinhos de kitting" - possam acessá-las e acondicioná-las em carrinhos específicos. O percurso realizado pelo carrinho dentro da área acontece através de trilhos. Cada carrinho é preenchido com uma coleção de peças de um mesmo veículo, que pode ser composta por itens de um ou mais postos de trabalho. A sua concepção é realizada com a intenção de atender toda a demanda de itens e peças, e de modo a permitir a mínima movimentação do operador da linha principal. Após a montagem, o carrinho é direcionado, através de trilhos RGVs (Rail Guided Vehicle) ou com o suporte de AGVs (Automated Guided Vehicle), para a entrada na linha, onde acompanhará o veículo até o final da extensão do seu respectivo tronçon.

As embalagens das diversas referências de itens e peças são basicamente divididas em dois tipos: embalagens pequenas (PE) com dimensões inferiores a 1000mm, 
armazenadas em móveis gravitacionais, aqui chamados de flowracks; e, em embalagens grandes, armazenadas em demarcações no piso.

Para o armazenamento de PEs, nas AK, são utilizados os flowracks, com prateleiras confeccionadas com pequenos rolos, não motorizados, que permitem a movimentação das embalagens por gravidade. A Figura 2 representa a visão lateral de um flowrack, já a Figura 3 ilustra um flowrack sendo utilizado na AK.

Figura 2 - Visão lateral de um flowrack.

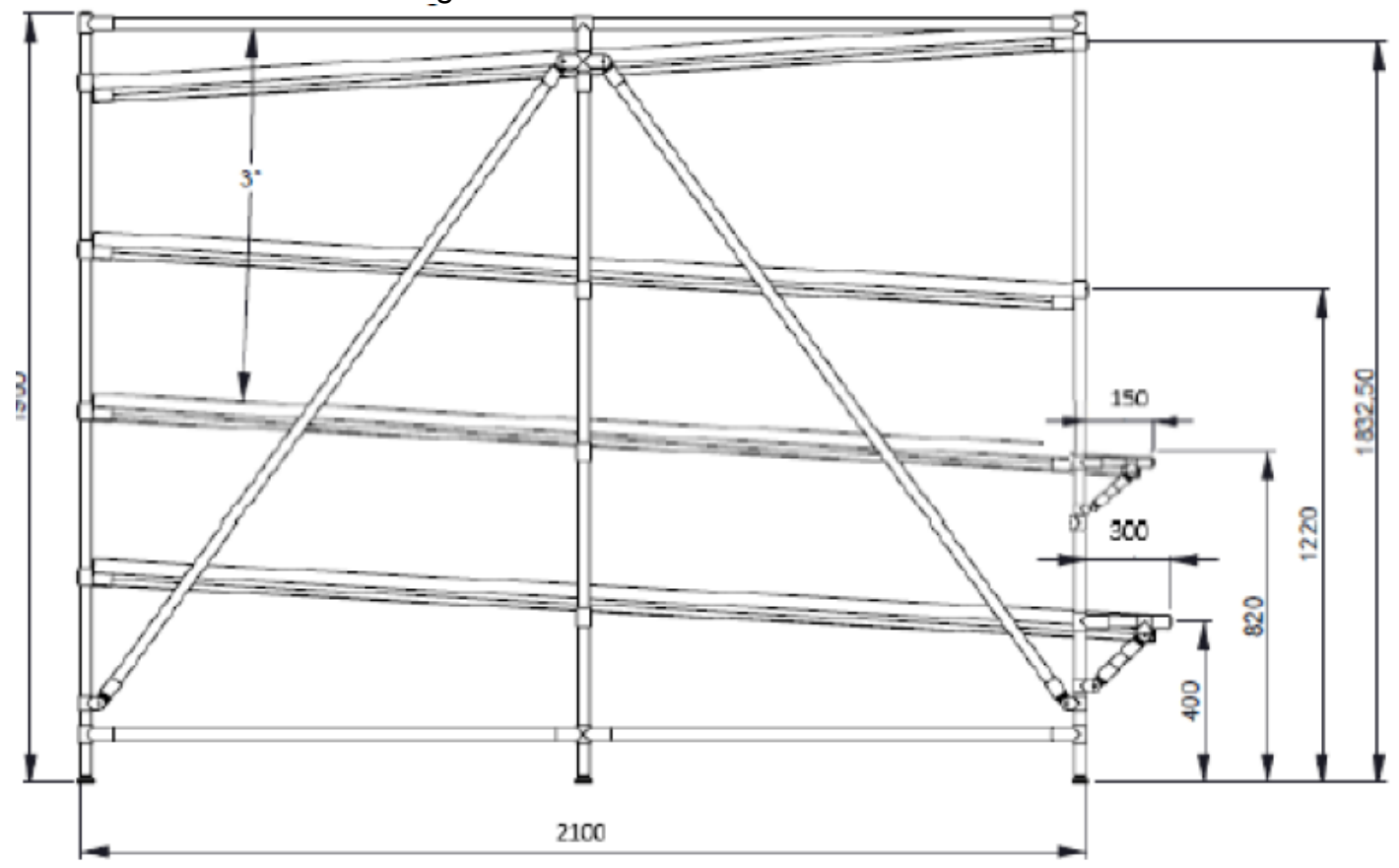

Fonte: Empresa ABC (2016). 
Figura 3 - Flowrack na AK.

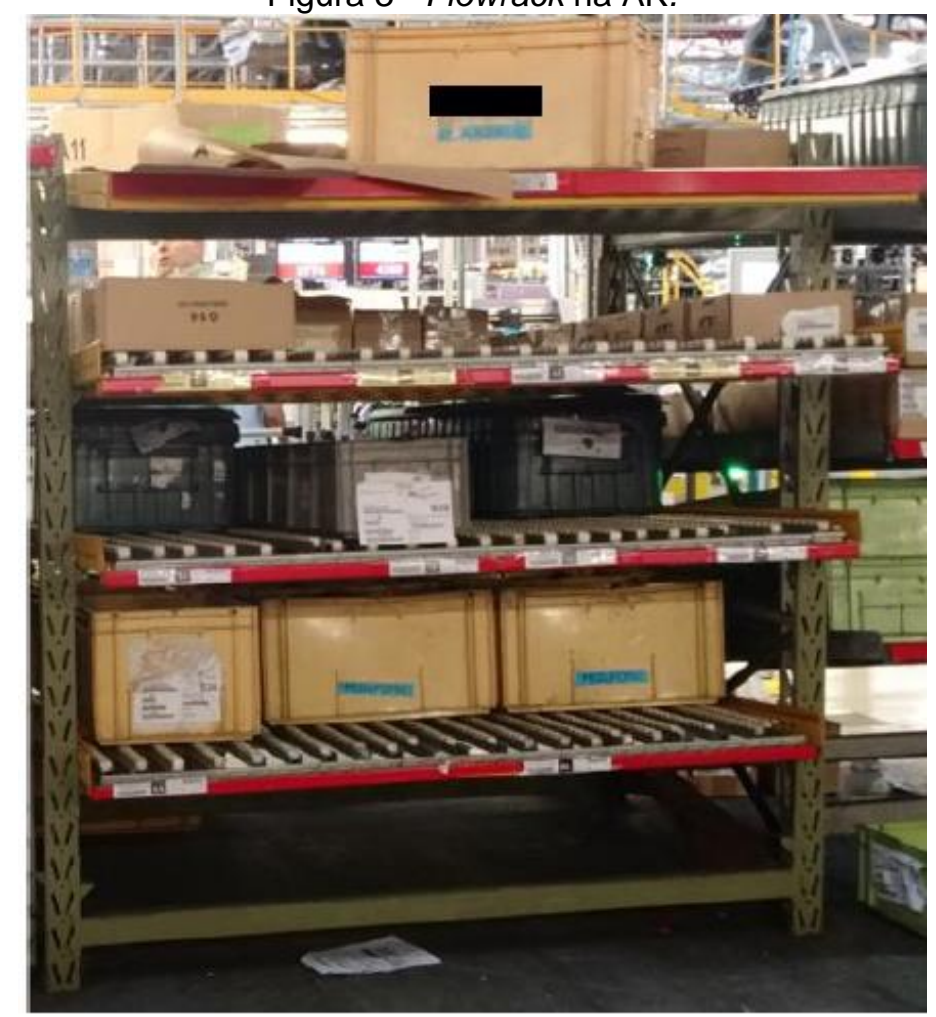

Fonte: Empresa ABC (2016).

\section{METODOLOGIA}

Conforme comentado anteriormente, pretendeu-se fazer o uso do algoritmo FFD para a otimização da alocação das PEs nos Flowracks existentes em cada AK. O método foi aplicado através do software Visual Basic for Applications ${ }^{\circledR}$ e executados em um notebook provido com processador Intel Core $^{\mathrm{TM}}$ 17-5500U de 2,4GHz, 16GB de memória RAM, e Windows 10 Home como sistema operacional.

As dimensões utilizadas para um flowrack foram 1900mmx1850mmx2100mm (altura $\mathrm{x}$ largura $x$ profundidade), subdividido em três prateleiras. Logo, para cada prateleira, tida como bin, foi empregado a dimensão de $1850 \mathrm{~mm}$. Já para as referências de peças, consideradas como itens, foi adotada a menor dimensão da embalagem entre largura e comprimento.

As $20 \mathrm{AKs}$, nas quais o algoritmo foi aplicado, possuem nomes específicos dentro da empresa. Neste estudo, foram denominadas de "AKXX" onde os valores de "XX" representam um número de identificação. Os flowracks atuais foram listados como " $F$ ", seguido de um número sequencial. A Tabela 1 apresenta as AK com as respectivas quantidades de referências e flowracks atuais. 
Tabela 1 - Quantidades de flowracks e referências de cada AK.

\begin{tabular}{cccc}
\hline ÁREA DE & \multicolumn{3}{c}{ QUANTIDADES } \\
\cline { 2 - 4 } KITIING & FLOWRACKS & PRATELEIRAS REFERÊNCIAS \\
\hline AK01 & 3 & 9 & 26 \\
AK02 & 1 & 3 & 14 \\
AK03 & 4 & 12 & 15 \\
AK04 & 5 & 15 & 30 \\
AK05 & 4 & 12 & 52 \\
AK06 & 2 & 6 & 19 \\
AK07 & 8 & 24 & 84 \\
AK08 & 15 & 45 & 146 \\
AK09 & 13 & 39 & 112 \\
AK10 & 2 & 6 & 9 \\
AK11 & 12 & 36 & 194 \\
AK12 & 11 & 33 & 104 \\
AK13 & 4 & 12 & 14 \\
AK14 & 1 & 3 & 9 \\
AK15 & 10 & 30 & 156 \\
AK16 & 13 & 39 & 124 \\
AK17 & 21 & 63 & 208 \\
AK18 & 2 & 6 & 18 \\
AK19 & 2 & 6 & 7 \\
AK20 & 1 & 3 & 14 \\
\hline
\end{tabular}

Fonte: Empresa ABC (2016).

A Tabela 2 exemplifica um caso de alocação atual em flowracks de todas as referências de PEs de uma AK, neste caso a área AK03. 
Tabela 2 - Exemplo de posição atual de PEs em flowracks.

\begin{tabular}{ccccccc}
\hline \multirow{2}{*}{ REFÊRENCIAS } & ÁREA DE & POSIÇÃO & \multicolumn{4}{c}{ DIMENSÕES } \\
\cline { 5 - 7 } & KITTING & ATUAL & ALTURA LARGURA COMPRIMENTO & MENOR \\
\hline REF1 & AK04 & F03 & 200 & 300 & 400 & 300 \\
REF2 & AK04 & F05 & 214 & 297 & 396 & 297 \\
REF3 & AK04 & F02 & 300 & 400 & 600 & 400 \\
REF4 & AK04 & F03 & 300 & 400 & 600 & 400 \\
REF5 & AK04 & F05 & 300 & 400 & 600 & 400 \\
REF6 & AK04 & F05 & 300 & 400 & 600 & 400 \\
REF7 & AK04 & F05 & 300 & 400 & 600 & 400 \\
REF8 & AK04 & F04 & 300 & 400 & 600 & 400 \\
REF9 & AK04 & F04 & 300 & 400 & 600 & 400 \\
REF10 & AK04 & F05 & 314 & 396 & 594 & 396 \\
REF11 & AK04 & F01 & 314 & 396 & 594 & 396 \\
REF12 & AK04 & F03 & 214 & 396 & 594 & 396 \\
REF13 & AK04 & F03 & 214 & 396 & 594 & 396 \\
REF14 & AK04 & F04 & 200 & 300 & 400 & 300 \\
REF15 & AK04 & F05 & 200 & 300 & 400 & 300 \\
REF16 & AK04 & F01 & 200 & 300 & 400 & 300 \\
REF17 & AK04 & F04 & 214 & 396 & 594 & 396 \\
REF18 & AK04 & F05 & 214 & 396 & 594 & 396 \\
REF19 & AK04 & F05 & 200 & 300 & 400 & 300 \\
REF20 & AK04 & F05 & 314 & 396 & 594 & 396 \\
REF21 & AK04 & F01 & 200 & 300 & 400 & 300 \\
REF22 & AK04 & F03 & 300 & 400 & 600 & 400 \\
REF23 & AK04 & F05 & 300 & 400 & 600 & 400 \\
REF24 & AK04 & F05 & 300 & 400 & 600 & 400 \\
REF25 & AK04 & F03 & 300 & 400 & 600 & 400 \\
REF26 & AK04 & F01 & 314 & 396 & 594 & 396 \\
REF27 & AK04 & F05 & 314 & 396 & 594 & 396 \\
REF28 & AK04 & F04 & 314 & 396 & 594 & 396 \\
REF29 & AK04 & F03 & 300 & 400 & 600 & 400 \\
REF30 & AK04 & F05 & 300 & 400 & 600 & 400 \\
\hline
\end{tabular}

Fonte: Empresa ABC (2016).

\section{RESULTADOS}

Após submeter os dados de todas as AK à implementação do algoritmo, obteve-se os valores apresentados na Tabela 3. 
Tabela 3 - Resultados comparativos após a implementação do método.

\begin{tabular}{cccccc}
\hline ÁREA DE & QUANTIDADE DE & \multicolumn{2}{c}{ QUANTIDADES ATUAIS } & \multicolumn{2}{c}{ QUANTIDADES FFD } \\
\cline { 3 - 6 } KITTING & REFERÊNCIAS & FLOWRACKS & PRATELEIRAS & FLOWRACKS PRATELEIRAS \\
\hline AK01 & 26 & 3 & 9 & 2 & 5 \\
AK02 & 14 & 1 & 3 & 1 & 3 \\
AK03 & 15 & 4 & 12 & 5 & 15 \\
AK04 & 30 & 5 & 15 & 2 & 6 \\
AK05 & 52 & 4 & 12 & 4 & 11 \\
AK06 & 19 & 2 & 6 & 2 & 5 \\
AK07 & 84 & 8 & 24 & 6 & 17 \\
AK08 & 146 & 15 & 45 & 10 & 30 \\
AK09 & 112 & 13 & 39 & 10 & 28 \\
AK10 & 9 & 2 & 6 & 1 & 3 \\
AK11 & 194 & 12 & 36 & 14 & 41 \\
AK12 & 104 & 11 & 33 & 9 & 26 \\
AK13 & 14 & 4 & 12 & 1 & 3 \\
AK14 & 9 & 1 & 3 & 1 & 3 \\
AK15 & 156 & 10 & 30 & 12 & 36 \\
AK16 & 124 & 13 & 39 & 9 & 27 \\
AK17 & 208 & 21 & 63 & 16 & 47 \\
AK18 & 18 & 2 & 6 & 4 & 10 \\
AK19 & 7 & 2 & 6 & 1 & 2 \\
AK20 & 14 & 1 & 3 & 1 & 2 \\
TOTAL & $\mathbf{1 3 5 5}$ & $\mathbf{1 3 4}$ & $\mathbf{4 0 2}$ & $\mathbf{1 1 1}$ & $\mathbf{3 2 0}$ \\
\hline
\end{tabular}

Fonte: autora (2016).

Na coluna apresentada como "Quantidades Atuais" é possível verificar as quantidades relativas aos flowracks e prateleiras existentes atualmente em cada AK. Já na coluna "Quantidade FFD", na subdivisão "Prateleiras", são apresentados os resultados obtidos a partir da execução do método, uma vez que cada unidade de prateleira é considerada como bin no problema. Para a obtenção dos valores da subdivisão flowracks, dividiu-se o número obtido de prateleiras pela capacidade de três prateleiras por unidade de flowrack.

Apresenta-se, na Tabela 4, os valores obtidos a partir da comparação entre as condições atuais de cada AK e os resultados da otimização. Na área AR04, por exemplo, houve a redução de 3 flowracks ou 9 prateleiras, resultando em $60 \%$ de diminuição na quantidade total. 
Tabela 4 - Redução entre valores atuais e o resultado da otimização.

\begin{tabular}{|c|c|c|c|c|}
\hline \multirow{2}{*}{$\begin{array}{l}\text { ÁREA DE } \\
\text { KITTING }\end{array}$} & \multicolumn{2}{|c|}{ REDUÇÃO (QUANTIDADE) } & \multicolumn{2}{|c|}{ REDUÇÃO (\%) } \\
\hline & FLOWRACKS & PRATELEIRAS & FLOWRACKS & PRATELEIRAS \\
\hline AK01 & 1 & 4 & $33,33 \%$ & $44,44 \%$ \\
\hline AK02 & 0 & 0 & $0,00 \%$ & $0,00 \%$ \\
\hline AK03 & -1 & -3 & $-25,00 \%$ & $-25,00 \%$ \\
\hline AK04 & 3 & 9 & $60,00 \%$ & $60,00 \%$ \\
\hline AK05 & 0 & 1 & $0,00 \%$ & $8,33 \%$ \\
\hline AK06 & 0 & 1 & $0,00 \%$ & $16,67 \%$ \\
\hline AK07 & 2 & 7 & $25,00 \%$ & $29,17 \%$ \\
\hline AK08 & 5 & 15 & $33,33 \%$ & $33,33 \%$ \\
\hline AK09 & 3 & 11 & $23,08 \%$ & $28,21 \%$ \\
\hline AK10 & 1 & 3 & $50,00 \%$ & $50,00 \%$ \\
\hline AK11 & -2 & -5 & $-16,67 \%$ & $-13,89 \%$ \\
\hline AK12 & 2 & 7 & $18,18 \%$ & $21,21 \%$ \\
\hline AK13 & 3 & 9 & $75,00 \%$ & $75,00 \%$ \\
\hline AK14 & 0 & 0 & $0,00 \%$ & $0,00 \%$ \\
\hline AK15 & -2 & -6 & $-20,00 \%$ & $-20,00 \%$ \\
\hline AK16 & 4 & 12 & $30,77 \%$ & $30,77 \%$ \\
\hline AK17 & 5 & 16 & $23,81 \%$ & $25,40 \%$ \\
\hline AK18 & -2 & -4 & $-100,00 \%$ & $-66,67 \%$ \\
\hline AK19 & 1 & 4 & $50,00 \%$ & $66,67 \%$ \\
\hline AK20 & 0 & 1 & $0,00 \%$ & $33,33 \%$ \\
\hline TOTAL & 23 & 82 & $17,16 \%$ & $20,40 \%$ \\
\hline
\end{tabular}

Fonte: autora (2016).

Os valores obtidos para as áreas AK03, AK11, AK15 e AK18 foram superiores aos encontrados atualmente, representando um aumento de $25 \%, 16,67 \%, 20 \%$ e $100 \%$ respectivamente, na quantidade total de flowracks. Dessa forma, essas áreas retratam os $22,2 \%$ mencionados por [13], em que o algoritmo se desvia do valor ótimo.

Tomando-se como exemplo a AK04, a Tabela 5 aponta a taxa de utilização (\%) e a sobra, em milímetros, de cada prateleira. Já, na Tabela 6 , é retratado a comparação entre a posição atual de determinada referência nos flowracks e a posição ótima encontrada pelo algoritmo. Ao considerar a redução dos 23 flowracks nas 20 áreas analisadas é possível gerar $89,36 \mathrm{~m}^{2}$ de área livre total nas AKs.

Tabela 5 - Valores de sobra e taxa de utilização das prateleiras.

\begin{tabular}{ccc}
\hline $\begin{array}{c}\text { PRATELEIRA } \\
(\text { bin })\end{array}$ & $\begin{array}{c}\text { SOBRA } \\
(\mathbf{m m})\end{array}$ & $\begin{array}{c}\text { TAXA DE } \\
\text { UTILIZAÇÃO }\end{array}$ \\
\hline 1 & 50 & $97 \%$ \\
2 & 66 & $96 \%$ \\
3 & 54 & $97 \%$ \\
4 & 50 & $97 \%$ \\
5 & 56 & $97 \%$ \\
6 & 1256 & $32 \%$ \\
\hline
\end{tabular}

Fonte: autora (2016). 
Tabela 6 - Comparação entre o posicionamento atual e ótimo das referências.

\begin{tabular}{cccc} 
REFERÊNCIAS & LOCAL & $\begin{array}{c}\text { POSIÇÃO } \\
\text { ATUAL }\end{array}$ & $\begin{array}{c}\text { POSIÇÃO } \\
\text { ÓIMA }\end{array}$ \\
\hline REF01 & AK04 & F03 & F01 \\
REF02 & AK04 & F05 & F02 \\
REF03 & AK04 & F02 & F01 \\
REF04 & AK04 & F03 & F01 \\
REF05 & AK04 & F05 & F02 \\
REF06 & AK04 & F05 & F01 \\
REF07 & AK04 & F05 & F01 \\
REF08 & AK04 & F04 & F01 \\
REF09 & AK04 & F04 & F01 \\
REF10 & AK04 & F05 & F01 \\
REF11 & AK04 & F01 & F01 \\
REF12 & AK04 & F03 & F01 \\
REF13 & AK04 & F03 & F02 \\
REF14 & AK04 & F04 & F02 \\
REF15 & AK04 & F05 & F02 \\
REF16 & AK04 & F01 & F01 \\
REF17 & AK04 & F04 & F01 \\
REF18 & AK04 & F05 & F02 \\
REF19 & AK04 & F05 & F02 \\
REF20 & AK04 & F05 & F03 \\
REF21 & AK04 & F01 & F01 \\
REF22 & AK04 & F03 & F02 \\
REF23 & AK04 & F05 & F02 \\
REF24 & AK04 & F05 & F02 \\
REF25 & AK04 & F03 & F02 \\
REF26 & AK04 & F01 & F01 \\
REF27 & AK04 & F05 & F02 \\
REF28 & AK04 & F04 & F01 \\
REF29 & AK04 & F03 & F01 \\
REF30 & AK04 & F05 & F02 \\
\hline & Fonte: autora (2016). & \\
\hline
\end{tabular}

Por fim, na Tabela 7, é apresentado o impacto em área a partir da redução da quantidade de flowracks proposta pelo FFD. 
Tabela 7 - Ganhos em área com a aplicação do método.

\begin{tabular}{cc}
\hline $\begin{array}{c}\text { ÁREA DE } \\
\text { KITTING }\end{array}$ & $\begin{array}{c}\text { GANHO DE ÁREA } \\
\left(\mathbf{m}^{\mathbf{2}}\right)\end{array}$ \\
\hline AK01 & 3,89 \\
AK02 & 0,00 \\
AK03 & $\mathbf{- 3 , 8 9}$ \\
AK04 & 11,66 \\
AK05 & 0,00 \\
AK06 & 0,00 \\
AK07 & 7,77 \\
AK08 & 19,43 \\
AK09 & 11,66 \\
AK10 & 3,89 \\
AK11 & $\mathbf{- 7 , 7 7}$ \\
AK12 & 7,77 \\
AK13 & 11,66 \\
AK14 & 0,00 \\
AK15 & $\mathbf{- 7 , 7 7}$ \\
AK16 & 15,54 \\
AK17 & 19,43 \\
AK18 & $\mathbf{- 7 , 7 7}$ \\
AK19 & 3,89 \\
AK20 & 0,00 \\
TOTAL & $\mathbf{8 9 , 3 6}$ \\
\hline F0nte
\end{tabular}

Fonte: autora (2016).

\section{CONCLUSÃO}

Neste estudo foi proposta a aplicação do algoritmo FFD como método de resolução para o BPP, a fim de resolver um problema aplicado a uma empresa automotiva. Buscou-se otimizar a alocação de referências de peças em flowracks, de forma a minimizar as quantidades e, assim, reduzir a área disponibilizada para a alocação dos mesmos.

O FFD é um método de fácil e rápida implementação computacional, porém com soluções razoáveis se comparado a outros métodos. Dessa forma, foi possível obter uma diminuição total de 23 flowracks, o que representa uma redução de $17,16 \%$ da necessidade destes, ao se analisar 20 AKs, resultando em $89,36 \mathrm{~m}^{2}$ de ganho de área somente com a redução deste meio.

Como sugestão de trabalhos futuros, tem-se a possibilidade da aplicação de outros métodos, heurísticos e meta-heurísticos, a fim de possibilitar uma comparação de resultados. 


\section{REFERÊNCIAS}

[1] ANFAVEA. Anuário da Indústria Automobilística Brasileira. Disponível em: http://www.anfavea.com.br/anuario.html. Acesso em: 14 de maio de 2016

[2] FENABRAVE. Índices e números de emplacamento de Janeiro/16. Disponível em: http://www3.fenabrave.org.br:8082/plus/modulos/listas/index.php?tac=indices-enumeros\&idtipo=1\&layout=indices-e-umeros. Acesso em: 14 de maio de 2016.

[3] ALOISE, D. J. Contribuições à solução do problema bin-packing: formulações, relaxações e novos algoritmos aproximativos. 165 p., Tese (Doutorado em Ciências em Engenharia de Sistemas e Computação) - Universidade Federal do Rio de Janeiro, RJ, 1992.

[4] SHWERIN, P.; WÄSCHER, G. The bin-packing problem: a problem generator and some numerical experiments with FFD packing and MTP , International Transactions in Operational Research, v. 4, p. 377-389, 1997.

[5] DYCKHOFF, H. A typology of cutting and packing problems, European Journal of Operational Research, v. 44, p. 145-159, 1990.

[6] JUNQUEIRA, N. M. P. Algoritmos aproximados para solucionar o problema de bin packing unidimensional, 137 p., Dissertação (Mestrado em Ciências) - Instituto Tecnológico de Aeronáutica, SP, 2007.

[7] COFFMAN JR., E.G.; GAREY, M.R.; JOHNSON, D.S. Approximation algorithms for bin-packing: a survey. In: Hochbaum, D., editor, APPROXIMATION ALGORITHMS FOR NP-HARD PROBLEMS, p. 46-93. PWS Publishing, 1997.

[8] KANTOROVICH, L. V. Mathematical methods of organizing and planning production, Management Science, v.6, p. 366-422, 1960.

[9] ALMEIDA, R. Proposta de um método meta-heurístico híbrido para resolução de problemas do tipo bin packing, 114 p., Dissertação (Mestrado em Engenharia de Produção) - Pontifícia Universidade Católica do Paraná, PR, 2014.

[10] MARTELLO, S.; TOTH, P. Knapsack problems: algorithms and computer implementations. John Wiley \& Sons, Cichester, 1990.

[11] EILON, S.; CHRISTOFIDES, N. The loading problem, Management Science, v.17, p. 259-268, 1971.

[12] HUNG. M.; BROWN, J. Na algorithm for a class of loading problems, Naval Research Logistics Quarterly, v.17, p. 289-297, 1978.

[13] SCHOLL, A.; KLEIN, R.; JÜRGENS, C. BISON: a fast hybrid procedure for exactly solving the one-dimensional bin packing problem, Computers Operations Research, v. 24, p. 627-645, 1997.

[14] DOKEROGLU, T.; COSAR, A. Optimization of one-dimensional bin packing problem with island. Parallel grouping genetic algorithms, Computers \& Industrial Engineering, v. 75, p. 176-186, 2014.

[15] BALAS, E. Na additive algorithm for solving linear programs with zero-one variables, Operations Research, v. 13, p. 517546, 1965.

[16] ALVIN, A. C. F Uma heurística híbrida de melhoria para o problema de bin packing pae sua aplicação ao problema de escalonamento de tarefas, 136 p., Tese (Doutorado em Informática) - Pontifícia Universidade Católica do Rio de Janeiro, RJ, 2003. 
[17] BRANDÃO, F.; PEDROSO, J. P. Bin packing and related problems: general arc-flow formulation with graph compression, Computers Operations Research, v. 69, p. 5667, 2016.

[18] GILMORE, P. C.; GOMORY, R. E. A linear programming approach to the cuttingstock problem, Operations Research, v. 9, p. 849-859, 1961. 